\title{
INEQUALITIES FOR THE RIEMANN-STIELTJES INTEGRAL OF $S$-DOMINATED INTEGRATORS WITH APPLICATIONS. I
}

Abstract. Assume that $u, v:[a, b] \rightarrow \mathbb{R}$ are monotonic nondecreasing on the interval $[a, b]$. We say that the complex-valued function $h:[a, b] \rightarrow \mathbb{C}$ is $S$-dominated by the pair $(u, v)$ if

$$
|h(y)-h(x)|^{2} \leq[u(y)-u(x)][v(y)-v(x)]
$$

for any $x, y \in[a, b]$. In this paper we show amongst other that

$$
\left|\int_{a}^{b} f(t) d h(t)\right|^{2} \leq \int_{a}^{b}|f(t)| d u(t) \int_{a}^{b}|f(t)| d v(t)
$$

for any continuous function $f:[a, b] \rightarrow \mathbb{C}$. Applications for the trapezoidal and midpoint inequalities are given. New inequalities for some Čebyšev and (CBS)-type functionals are presented. Natural applications for continuous functions of selfadjoint and unitary operators on Hilbert spaces are provided as well.

Key words: Riemann-Stieltjes integral, functions of bounded variation, cumulative variation, selfadjoint operators, unitary operators, trapezoid and midpoint inequalities, Čebyšev and (CBS)type functionals

2010 Mathematical Subject Classification: 26D15, 47A63

1. Introduction. One of the most important properties of the Riemann-Stieltjes integral $\int_{a}^{b} f(t) d g(t)$ is the fact that this integral exists if one of the functions is of bounded variation while the other is continuous. The following sharp inequality holds

$$
\left|\int_{a}^{b} f(t) d g(t)\right| \leq \max _{t \in[a, b]}|f(t)| \bigvee_{a}^{b}(g),
$$

(C) Petrozavodsk State University, 2015 
provided that $f:[a, b] \rightarrow \mathbb{C}$ is continuous on $[a, b]$ and $g:[a, b] \rightarrow \mathbb{C}$ is of bounded variation on this interval. Here $\bigvee_{a}^{b}(g)$ denotes the total variation of $g$ on $[a, b]$.

When $g$ is Lipschitzian with the constant $L>0$, i.e.,

$$
|g(t)-g(s)| \leq L|t-s|
$$

for any $t, s \in[a, b]$, then we have

$$
\left|\int_{a}^{b} f(t) d g(t)\right| \leq L \int_{a}^{b}|f(t)| d t
$$

for any Riemann integrable function $f:[a, b] \rightarrow \mathbb{C}$.

Moreover, if the integrator $g$ is monotonic nondecreasing on the interval $[a, b]$ and $f:[a, b] \rightarrow \mathbb{C}$ is continuous, then we have the modulus inequality

$$
\left|\int_{a}^{b} f(t) d g(t)\right| \leq \int_{a}^{b}|f(t)| d g(t) .
$$

The above inequalities have been used by many authors to derive various integral inequalities. We provide here some simple examples.

The following generalized trapezoidal inequality for the function of bounded variation $f:[a, b] \rightarrow \mathbb{C}$ was obtained in 1999 by the author [1, Proposition 1]

$$
\begin{aligned}
& \left|\int_{a}^{b} f(t) d t-(x-a) f(a)-(b-x) f(b)\right| \leq \\
& \leq\left[\frac{1}{2}(b-a)+\left|x-\frac{a+b}{2}\right|\right] \bigvee_{a}^{b}(f),
\end{aligned}
$$

where $x \in[a, b]$. The constant $\frac{1}{2}$ cannot be replaced by a smaller quantity. See also [2] for a different proof and other details.

The best inequality one can derive from (1) is the trapezoid inequality

$$
\left|\int_{a}^{b} f(t) d t-\frac{f(a)+f(b)}{2}(b-a)\right| \leq \frac{1}{2}(b-a) \bigvee_{a}^{b}(f) .
$$

Here the constant $\frac{1}{2}$ is also the best possible. 
For related results, see [3]-27.

In order to extend the classical Ostrowski's inequality for differentiable functions with bounded derivatives to the larger class of functions of bounded variation, the author obtained in 1999 (see [1] or the RGMIA preprint version of [28]) the following result

$$
\left|\int_{a}^{b} f(t) d t-f(x)(b-a)\right| \leq\left[\frac{1}{2}(b-a)+\left|x-\frac{a+b}{2}\right|\right] \bigvee_{a}^{b}(f),
$$

for any $x \in[a, b]$ and $f:[a, b] \rightarrow \mathbb{C}$ is a function of bounded variation on $[a, b]$. Here $\bigvee_{a}^{b}(f)$ denotes the total variation of $f$ on $[a, b]$ and the constant $\frac{1}{2}$ is the best possible in $(2)$. The best inequality one can obtain from (2) is the midpoint inequality, namely

$$
\left|\int_{a}^{b} f(t) d t-f\left(\frac{a+b}{2}\right)(b-a)\right| \leq \frac{1}{2}(b-a) \bigvee_{a}^{b}(f),
$$

for which the constant $\frac{1}{2}$ is also sharp.

For related results, see [29]-[57].

Motivated by the above results, we establish in this paper a bound for the quantity

$$
\left|\int_{a}^{b} f(t) d g(t)\right|
$$

in the case when the integrand $f$ is continuous while the function of bounded variation $g$ is $S$-dominated by a pair of monotonic functions in the sense presented at the beginning of the next section. The applications for the trapezoidal and midpoint inequalities are given. New inequalities for some Čebyšev and (CBS)-type functionals are presented. Natural applications for continuous functions of selfadjoint and unitary operators on Hilbert spaces are provided as well.

2. Some General Inequalities. Assume that $u, v:[a, b] \rightarrow \mathbb{R}$ are monotonic nondecreasing on the interval $[a, b]$. We say that the complexvalued function $h:[a, b] \rightarrow \mathbb{C}$ is $S$-dominated by the pair $(u, v)$ if

$$
|h(y)-h(x)|^{2} \leq[u(y)-u(x)][v(y)-v(x)]
$$

for any $x, y \in[a, b]$. 
We observe that by the monotonicity of the functions $u$ and $v$ and by the symmetry of the inequality $(\mathrm{S})$ over $x$ and $y$ we can assume that $(\mathrm{S})$ is satisfied only for $y>x$ with $x, y \in[a, b]$.

We can give numerous examples of such functions.

For instance, if we take $f, g \in L_{2}[a, b]$, where $L_{2}[a, b]$ is the Hilbert space of all complex-valued functions that are square-Lebesgue integrable, and denote

$h(x):=\int_{a}^{x} f(t) g(t) d t, u(x):=\int_{a}^{x}|f(t)|^{2} d t$ and $v(x):=\int_{a}^{x}|g(t)|^{2} d t$,

then we observe that $u$ and $v$ are monotonic nondecreasing on $[a, b]$ and by Cauchy-Bunyakovsky-Schwarz integral inequality we have

$$
\begin{aligned}
|h(y)-h(x)|^{2} & =\left|\int_{x}^{y} f(t) g(t) d t\right|^{2} \leq \int_{x}^{y}|f(t)|^{2} d t \int_{x}^{y}|g(t)|^{2} d t \leq \\
& \leq[u(y)-u(x)][v(y)-v(x)] .
\end{aligned}
$$

for any $y>x$ with $x, y \in[a, b]$.

Now, for $p, q>0$ if we consider $f(t):=t^{p}$ and $g(t):=t^{q}$ for $t \geq 0$, then

$$
h_{p, q}(x):=\int_{0}^{x} t^{p+q} d t=\frac{1}{p+q+1} x^{p+q+1}
$$

and

$$
u_{p}(x):=\int_{0}^{x} t^{2 p} d t=\frac{1}{2 p+1} x^{2 p+1}, v_{q}(x):=\int_{0}^{x} t^{2 q} d t=\frac{1}{2 q+1} x^{2 q+1} .
$$

Taking into account the above comments we observe that the function $h_{p, q}$ is $S$-dominated by the pair $\left(u_{p}, v_{q}\right)$ on any subinterval of $[0, \infty)$.

Proposition 1. If $h:[a, b] \rightarrow \mathbb{C}$ is $S$-dominated by the pair $(u, v)$, then $h$ is of bounded variation on any subinterval $[c, d] \subset[a, b]$ and

$$
\left[\bigvee_{c}^{d}(h)\right]^{2} \leq[u(d)-u(c)][v(d)-v(c)]
$$

Proof. Consider a division $\delta$ of the interval $[c, d]$ given by

$$
\delta: c=x_{0}<x_{1}<\ldots<x_{n-1}<x_{n}=b .
$$


Since $h:[a, b] \rightarrow \mathbb{C}$ is $\mathrm{S}$-dominated by the pair $(u, v)$ then we have

$$
\left|h\left(x_{i+1}\right)-h\left(x_{i}\right)\right| \leq\left[u\left(x_{i+1}\right)-u\left(x_{i}\right)\right]^{1 / 2}\left[v\left(x_{i+1}\right)-v\left(x_{i}\right)\right]^{1 / 2}
$$

for any $i \in\{0, \ldots, n-1\}$.

Summing this inequality over $i$ from 0 to $n-1$ and utilizing the Cauchy-Bunyakovsky-Schwarz discrete inequality we have

$$
\begin{aligned}
& \sum_{i=1}^{n-1}\left|h\left(x_{i+1}\right)-h\left(x_{i}\right)\right| \leq \\
& \leq \sum_{i=1}^{n-1}\left[u\left(x_{i+1}\right)-u\left(x_{i}\right)\right]^{1 / 2}\left[v\left(x_{i+1}\right)-v\left(x_{i}\right)\right]^{1 / 2} \leq \\
& \leq\left(\sum_{i=1}^{n-1}\left[u\left(x_{i+1}\right)-u\left(x_{i}\right)\right]\right)^{1 / 2}\left(\sum_{i=1}^{n-1}\left[v\left(x_{i+1}\right)-v\left(x_{i}\right)\right]\right)^{1 / 2}= \\
& =[u(d)-u(c)]^{1 / 2}[v(d)-v(c)]^{1 / 2} .
\end{aligned}
$$

Taking the supremum over $\delta$ we deduce the desired result (3).

Corollary 1. If $h:[a, b] \rightarrow \mathbb{C}$ is $S$-dominated by the pair $(u, v)$, then the cumulative variation function $V:[a, b] \rightarrow[0, \infty)$ defined by

$$
V(x):=\bigvee_{a}^{x}(h)
$$

is also $S$-dominated by the pair $(u, v)$.

Theorem 1. Assume that $u, v:[a, b] \rightarrow \mathbb{R}$ are monotonic nondecreasing on the interval $[a, b]$. If $h:[a, b] \rightarrow \mathbb{C}$ is $S$-dominated by the pair $(u, v)$ and $f:[a, b] \rightarrow \mathbb{C}$ is a continuous function on $[a, b]$, then the Riemann-Stieltjes integral $\int_{a}^{b} f(t) d h(t)$ exists and

$$
\left|\int_{a}^{b} f(t) d h(t)\right|^{2} \leq \int_{a}^{b}|f(t)| d u(t) \int_{a}^{b}|f(t)| d v(t) .
$$

Proof. Since the Riemann-Stieltjes integral $\int_{a}^{b} f(t) d h(t)$ exists, then for any sequence of partitions

$$
I_{n}^{(n)}: a=t_{0}^{(n)}<t_{1}^{(n)}<\cdots<t_{n-1}^{(n)}<t_{n}^{(n)}=b
$$


with the norm

$$
v\left(I_{n}^{(n)}\right):=\max _{i \in\{0, \ldots, n-1\}}\left(t_{i+1}^{(n)}-t_{i}^{(n)}\right) \rightarrow 0 \quad \text { as } \quad n \rightarrow \infty,
$$

and for any intermediate points $\xi_{i}^{(n)} \in\left[t_{i}^{(n)}, t_{i+1}^{(n)}\right], i \in\{0, \ldots, n-1\}$ we have:

$$
\begin{aligned}
& \left|\int_{a}^{b} f(t) d h(t)\right|= \\
& =\left|\lim _{v\left(I_{n}^{(n)}\right) \rightarrow 0} \sum_{i=0}^{n-1} f\left(\xi_{i}^{(n)}\right)\left[h\left(t_{i+1}^{(n)}\right)-h\left(t_{i}^{(n)}\right)\right]\right| \leq \\
& \leq \lim _{v\left(I_{n}^{(n)}\right) \rightarrow 0} \sum_{i=0}^{n-1}\left|f\left(\xi_{i}^{(n)}\right)\right|\left|h\left(t_{i+1}^{(n)}\right)-h\left(t_{i}^{(n)}\right)\right| \leq \\
& \leq \lim _{v\left(I_{n}^{(n)}\right) \rightarrow 0} \sum_{i=0}^{n-1}\left|f\left(\xi_{i}^{(n)}\right)\right|\left[u\left(t_{i+1}^{(n)}\right)-u\left(t_{i}^{(n)}\right)\right]^{\frac{1}{2}}\left[v\left(t_{i+1}^{(n)}\right)-v\left(t_{i}^{(n)}\right)\right]^{\frac{1}{2}} \leq \\
& \leq \lim _{v\left(I_{n}^{(n)}\right) \rightarrow 0}\left(\sum_{i=0}^{n-1}\left|f\left(\xi_{i}^{(n)}\right)\right|\left[u\left(t_{i+1}^{(n)}\right)-u\left(t_{i}^{(n)}\right)\right]\right)^{1 / 2} \times \\
& \times \lim _{v\left(I_{n}^{(n)}\right) \rightarrow 0}\left(\sum_{i=0}^{n-1}\left|f\left(\xi_{i}^{(n)}\right)\right|\left[v\left(t_{i+1}^{(n)}\right)-v\left(t_{i}^{(n)}\right)\right]\right)^{1 / 2}= \\
& =\left(\int_{a}^{b}|f(t)| d u(t)\right)^{1 / 2}\left(\int_{a}^{b}|f(t)| d v(t)\right)^{1 / 2},
\end{aligned}
$$

where for the last inequality we employed the Cauchy-BunyakovskySchwarz weighted discrete inequality

$$
\sum_{k=1}^{n} m_{k} a_{k} b_{k} \leq\left(\sum_{k=1}^{n} m_{k} a_{k}^{2}\right)^{1 / 2}\left(\sum_{k=1}^{n} m_{k} b_{k}^{2}\right)^{1 / 2},
$$

where $m_{k}, a_{k}, b_{k} \geq 0$ for $k \in\{1, \ldots, n\}$.

3. Trapezoid and Midpoint Inequalities. We can use the inequality (5) to derive various inequalities of trapezoidal and midpoint type as follows. 
Theorem 2. Assume that $u, v:[a, b] \rightarrow \mathbb{R}$ are monotonic nondecreasing on the interval $[a, b]$. If $h:[a, b] \rightarrow \mathbb{C}$ is $S$-dominated by the pair $(u, v)$, then

$$
\begin{aligned}
& \left|\frac{h(a)+h(b)}{2}(b-a)-\int_{a}^{b} h(t) d t\right|^{2} \leq \\
& \leq\left[\frac{1}{2}(b-a)[u(b)-u(a)]-\int_{a}^{b} \operatorname{sgn}\left(t-\frac{a+b}{2}\right) u(t) d t\right] \times \\
& \times\left[\frac{1}{2}(b-a)[v(b)-v(a)]-\int_{a}^{b} \operatorname{sgn}\left(t-\frac{a+b}{2}\right) v(t) d t\right] \leq \\
& \leq \frac{1}{4}(b-a)^{2}[u(b)-u(a)][v(b)-v(a)] .
\end{aligned}
$$

Proof. Integrating by parts in the Riemann-Stieltjes integral, we have

$$
\frac{h(a)+h(b)}{2}(b-a)-\int_{a}^{b} h(t) d t=\int_{a}^{b}\left(t-\frac{a+b}{2}\right) d h(t) .
$$

Applying the inequality (5) we have

$$
\left|\int_{a}^{b}\left(t-\frac{a+b}{2}\right) d h(t)\right|^{2} \leq \int_{a}^{b}\left|t-\frac{a+b}{2}\right| d u(t) \int_{a}^{b}\left|t-\frac{a+b}{2}\right| d v(t) .
$$

Integrating by parts in the Riemann-Stieltjes integral we also have

$$
\begin{aligned}
& \int_{a}^{b}\left|t-\frac{a+b}{2}\right| d u(t)= \\
& =\int_{a}^{\frac{a+b}{2}}\left(\frac{a+b}{2}-t\right) d u(t)+\int_{\frac{a+b}{2}}^{b}\left(t-\frac{a+b}{2}\right) d u(t)= \\
& =\left.\left(\frac{a+b}{2}-t\right) u(t)\right|_{a} ^{\frac{a+b}{2}}+\int_{a}^{\frac{a+b}{2}} u(t) d t+ \\
& +\left.\left(t-\frac{a+b}{2}\right) u(t)\right|_{\frac{a+b}{2}} ^{b}-\int_{\frac{a+b}{2}}^{b} u(t) d t= \\
& =-\frac{b-a}{2} u(a)+\int_{a}^{\frac{a+b}{2}} u(t) d t+\frac{b-a}{2} u(b)-\int_{\frac{a+b}{2}}^{b} u(t) d t=
\end{aligned}
$$




$$
=\frac{1}{2}(b-a)[u(b)-u(a)]-\int_{a}^{b} \operatorname{sgn}\left(t-\frac{a+b}{2}\right) u(t) d t
$$

and a similar relation for $v$.

By the Čebyšev inequality for monotonic nondecreasing functions $F$, $G$ that states that

$$
\frac{1}{b-a} \int_{a}^{b} F(t) G(t) d t \geq \frac{1}{b-a} \int_{a}^{b} F(t) d t \cdot \frac{1}{b-a} \int_{a}^{b} G(t) d t
$$

we also have

$$
\begin{aligned}
& \int_{a}^{b} \operatorname{sgn}\left(t-\frac{a+b}{2}\right) u(t) d t \geq \\
& \geq \frac{1}{b-a} \int_{a}^{b} \operatorname{sgn}\left(t-\frac{a+b}{2}\right) d t \int_{a}^{b} u(t) d t=0
\end{aligned}
$$

and a similar result for $v$.

Utilizing (8)-(11) we deduce the desired result (7).

Theorem 3. Assume that $u, v:[a, b] \rightarrow \mathbb{R}$ are monotonic nondecreasing on the interval $[a, b]$. If $h:[a, b] \rightarrow \mathbb{C}$ is $S$-dominated by the pair $(u, v)$, then

$$
\begin{aligned}
& \left|h\left(\frac{a+b}{2}\right)(b-a)-\int_{a}^{b} h(t) d t\right|^{2} \leq \\
& \leq \int_{a}^{b} \operatorname{sgn}\left(t-\frac{a+b}{2}\right) u(t) d t \int_{a}^{b} \operatorname{sgn}\left(t-\frac{a+b}{2}\right) v(t) d t \leq \\
& \leq \frac{1}{4}(b-a)^{2}[u(b)-u(a)][v(b)-v(a)] .
\end{aligned}
$$

Proof. Integrating by parts in the Riemann-Stieltjes integral we have

$$
\begin{aligned}
& h\left(\frac{a+b}{2}\right)(b-a)-\int_{a}^{b} h(t) d t= \\
& =\int_{a}^{\frac{a+b}{2}}(t-a) d h(t)-\int_{\frac{a+b}{2}}^{b}(b-t) d h(t) .
\end{aligned}
$$


Taking the modulus in 13 we have

$$
\begin{aligned}
& \left|h\left(\frac{a+b}{2}\right)(b-a)-\int_{a}^{b} h(t) d t\right| \leq \\
& \leq\left|\int_{a}^{\frac{a+b}{2}}(t-a) d h(t)\right|+\left|\int_{\frac{a+b}{2}}^{b}(b-t) d h(t)\right| .
\end{aligned}
$$

Applying the inequality (5) twice, we have

$$
\left|\int_{a}^{\frac{a+b}{2}}(t-a) d h(t)\right| \leq\left(\int_{a}^{\frac{a+b}{2}}(t-a) d u(t)\right)^{1 / 2}\left(\int_{a}^{\frac{a+b}{2}}(t-a) d v(t)\right)^{1 / 2}
$$

and

$$
\left|\int_{\frac{a+b}{2}}^{b}(b-t) d h(t)\right| \leq\left(\int_{\frac{a+b}{2}}^{b}(b-t) d u(t)\right)^{1 / 2}\left(\int_{\frac{a+b}{2}}^{b}(b-t) d v(t)\right)^{1 / 2} .
$$

Summing these inequalities and utilizing the elementary result

$$
\alpha \beta+\lambda \delta \leq\left(\alpha^{2}+\lambda^{2}\right)^{1 / 2}\left(\beta^{2}+\delta^{2}\right)^{1 / 2}
$$

where $\alpha, \beta, \lambda, \delta \geq 0$, we have

$$
\begin{aligned}
& \left|\int_{a}^{\frac{a+b}{2}}(t-a) d h(t)\right|+\left|\int_{\frac{a+b}{2}}^{b}(b-t) d h(t)\right| \leq \\
& \leq\left(\int_{a}^{\frac{a+b}{2}}(t-a) d u(t)\right)^{1 / 2}\left(\int_{a}^{\frac{a+b}{2}}(t-a) d v(t)\right)^{1 / 2}+ \\
& +\left(\int_{\frac{a+b}{2}}^{b}(b-t) d u(t)\right)^{1 / 2}\left(\int_{\frac{a+b}{2}}^{b}(b-t) d v(t)\right)^{1 / 2} \leq \\
& \leq\left(\int_{a}^{\frac{a+b}{2}}(t-a) d u(t)+\int_{\frac{a+b}{2}}^{b}(b-t) d u(t)\right)^{1 / 2}+ \\
& +\left(\int_{a}^{\frac{a+b}{2}}(t-a) d v(t)+\int_{\frac{a+b}{2}}^{b}(b-t) d v(t)\right)^{1 / 2} .
\end{aligned}
$$


Integrating by parts in the Riemann-Stieltjes integral we have

$$
\begin{aligned}
& \int_{a}^{\frac{a+b}{2}}(t-a) d u(t)+\int_{\frac{a+b}{2}}^{b}(b-t) d u(t)= \\
& =\left.(t-a) u(t)\right|_{a} ^{\frac{a+b}{2}}-\int_{a}^{\frac{a+b}{2}} u(t) d t+\left.(b-t) u(t)\right|_{\frac{a+b}{2}} ^{b}+\int_{\frac{a+b}{2}}^{b} u(t) d t= \\
& =\frac{1}{2}(b-a) u\left(\frac{a+b}{2}\right)-\int_{a}^{\frac{a+b}{2}} u(t) d t- \\
& -\frac{1}{2}(b-a) u\left(\frac{a+b}{2}\right)+\int_{\frac{a+b}{2}}^{b} u(t) d t= \\
& =\int_{a}^{b} \operatorname{sgn}\left(t-\frac{a+b}{2}\right) u(t) d t
\end{aligned}
$$

and the last integral is nonnegative as shown in the proof of Theorem 2 The same equality holds for $v$ as well.

Utilising the Grüss integral inequality

$$
\begin{aligned}
& \left|\frac{1}{b-a} \int_{a}^{b} F(t) G(t) d t-\frac{1}{b-a} \int_{a}^{b} F(t) d t \cdot \frac{1}{b-a} \int_{a}^{b} G(t) d t\right| \leq \\
& \leq \frac{1}{4}(M-m)(N-n)
\end{aligned}
$$

that holds for the Lebesgue integrable functions $F$ and $G$ that satisfy the conditions

$$
m \leq F(t) \leq M \text { and } n \leq G(t) \leq N
$$

for almost every $t \in[a, b]$, we have

$$
\begin{aligned}
0 & \leq \frac{1}{b-a} \int_{a}^{b} \operatorname{sgn}\left(t-\frac{a+b}{2}\right) u(t) d t= \\
& =\frac{1}{b-a} \int_{a}^{b} \operatorname{sgn}\left(t-\frac{a+b}{2}\right) u(t) d t- \\
& -\frac{1}{b-a} \int_{a}^{b} \operatorname{sgn}\left(t-\frac{a+b}{2}\right) d t \cdot \frac{1}{b-a} \int_{a}^{b} u(t) d t \leq \\
& \leq \frac{1}{2}[u(b)-u(a)]
\end{aligned}
$$


which implies that

$$
\int_{a}^{b} \operatorname{sgn}\left(t-\frac{a+b}{2}\right) u(t) d t \leq \frac{1}{2}(b-a)[u(b)-u(a)] .
$$

A similar result holds for $v$.

Making use of the inequalities (14), (15), 116) and (18) we deduce the desired result 12 .

\section{Applications for Čebyšev and (CBS)-Type Functionals.} The following lemma is of interest in itself.

Lemma 1. Let $F:[a, b] \times[a, b] \rightarrow \mathbb{C}$ be continuous on the rectangle $[a, b] \times[a, b]$ and let $h:[a, b] \rightarrow \mathbb{C}$ be an $S$-dominated function by the pair $(u, v)$ which are monotonic nondecreasing on $[a, b]$. Then we have

$$
\begin{aligned}
& \left|\int_{a}^{b}\left(\int_{a}^{b} F(x, y) d h(y)\right) d h(x)\right|^{2} \leq \\
& \leq\left(\int_{a}^{b}\left(\int_{a}^{b}|F(x, y)| d u(y)\right) d u(x)\right)^{1 / 2} \times \\
& \times\left(\int_{a}^{b}\left(\int_{a}^{b}|F(x, y)| d v(y)\right) d v(x)\right)^{1 / 2} \times \\
& \times\left(\int_{a}^{b}\left(\int_{a}^{b}|F(x, y)| d u(y)\right) d v(x)\right)^{1 / 2} \times \\
& \times\left(\int_{a}^{b}\left(\int_{a}^{b}|F(x, y)| d v(y)\right) d u(x)\right)^{1 / 2} .
\end{aligned}
$$

Proof. Assume that $x$ is fixed in $[a, b]$. If we apply Theorem 1 for the $S$-dominated function $h:[a, b] \rightarrow \mathbb{C}$ we have

$$
\left|\int_{a}^{b} F(x, y) d h(y)\right| \leq\left(\int_{a}^{b}|F(x, y)| d u(y)\right)^{1 / 2}\left(\int_{a}^{b}|F(x, y)| d v(y)\right)^{1 / 2} .
$$


Applying again Theorem 1 and utilizing (20) we have

$$
\begin{gathered}
\left|\int_{a}^{b}\left(\int_{a}^{b} F(x, y) d h(y)\right) d h(x)\right|^{2} \leq \\
\leq \int_{a}^{b}\left|\int_{a}^{b} F(x, y) d h(y)\right| d u(x) \int_{a}^{b}\left|\int_{a}^{b} F(x, y) d h(y)\right| d v(x) \leq \\
\leq \int_{a}^{b}\left(\int_{a}^{b}|F(x, y)| d u(y)\right)^{1 / 2}\left(\int_{a}^{b}|F(x, y)| d v(y)\right)^{1 / 2} d u(x) \times \\
\times \int_{a}^{b}\left(\int_{a}^{b}|F(x, y)| d u(y)\right)^{1 / 2}\left(\int_{a}^{b}|F(x, y)| d v(y)\right)^{1 / 2} d v(x) .
\end{gathered}
$$

On making use of the Cauchy-Bunyakovsky-Schwarz inequality for the Riemann-Stieltjes integral of monotonic nondecreasing integrators we have for the integrator $u$

$$
\begin{aligned}
& \int_{a}^{b}\left(\int_{a}^{b}|F(x, y)| d u(y)\right)^{1 / 2}\left(\int_{a}^{b}|F(x, y)| d v(y)\right)^{1 / 2} d u(x) \leq \\
& \leq\left(\int_{a}^{b}\left(\int_{a}^{b}|F(x, y)| d u(y)\right) d u(x)\right)^{1 / 2} \times \\
& \times\left(\left(\int_{a}^{b}|F(x, y)| d v(y)\right) d u(x)\right)^{1 / 2}
\end{aligned}
$$

and for the integrator $v$

$$
\begin{aligned}
& \int_{a}^{b}\left(\int_{a}^{b}|F(x, y)| d u(y)\right)^{1 / 2}\left(\int_{a}^{b}|F(x, y)| d v(y)\right)^{1 / 2} d v(x) \leq \\
& \leq\left(\int_{a}^{b}\left(\int_{a}^{b}|F(x, y)| d u(y)\right) d v(x)\right)^{1 / 2} \times \\
& \times\left(\left(\int_{a}^{b}|F(x, y)| d v(y)\right) d v(x)\right)^{1 / 2} .
\end{aligned}
$$

Utilising (21)-23) we deduce the desired result (19). 
When no confusion is possible, we write $\int_{a}^{b} f d u$ instead of $\int_{a}^{b} f(x) d u(x)$.

For the complex-valued functions $p, f, g$ and $h, \ell$ defined on the interval $[a, b]$ we define the following Čebyšev type functionals

$$
C(p, f, g ; h):=\int_{a}^{b} p d h \int_{a}^{b} p f g d h-\int_{a}^{b} p f d h \int_{a}^{b} p g d h
$$

and

$$
\begin{aligned}
C(p, f, g ; h, \ell) & =\int_{a}^{b} p d \ell \int_{a}^{b} p f g d h+\int_{a}^{b} p d h \int_{a}^{b} p f g d \ell- \\
& -\int_{a}^{b} p f d \ell \int_{a}^{b} p g d h-\int_{a}^{b} p f d h \int_{a}^{b} p g d \ell
\end{aligned}
$$

provided that all the Riemann-Stieltjes integrals involved above exist.

We observe that

$$
C(p, f, g ; h, h)=2 C(p, f, g ; h)
$$

and

$$
C(p, f, g ; h, \ell)=C(p, f, g ; \ell, h) .
$$

Theorem 4. Let $f, g:[a, b] \rightarrow \mathbb{R}$ be continuous and synchronous on $[a, b]$, i.e.,

$$
(f(x)-f(y))(g(x)-g(y)) \geq 0
$$

for any $x, y \in[a, b]$. If $h:[a, b] \rightarrow \mathbb{C}$ is an $S$-dominated function by the pair $(u, v)$ which are monotonic nondecreasing on $[a, b]$, then for any continuous nonnegative function $p:[a, b] \rightarrow[0, \infty)$ we have

$$
|C(p, f, g ; h)|^{2} \leq \frac{1}{2} C(p, f, g ; u, v)[C(p, f, g ; u)]^{1 / 2}[C(p, f, g ; v)]^{1 / 2} .
$$

Proof. Define the function $F:[a, b] \times[a, b] \rightarrow \mathbb{R}$ by

$$
\begin{aligned}
F(x, y) & :=p(x) p(y)(f(x)-f(y))(g(x)-g(y))= \\
& =p(y) p(x) f(x) g(x)+p(x) p(y) f(y) g(y)- \\
& -p(x) f(x) p(y) g(y)-p(y) f(y) p(x) g(x) .
\end{aligned}
$$

We observe that, since $p$ is nonnegative and $f, g$ are synchronous, then $F(x, y) \geq 0$ for any $x, y \in[a, b]$. The function $F$ is also continuous on the rectangle $[a, b] \times[a, b]$. 
By simple calculation with the Riemann-Stieltjes integral we have

$$
\begin{gathered}
\int_{a}^{b}\left(\int_{a}^{b} F(x, y) d h(y)\right) d h(x)=2 C(p, f, g ; h), \\
\int_{a}^{b}\left(\int_{a}^{b}|F(x, y)| d u(y)\right) d u(x)=2 C(p, f, g ; u) \geq 0, \\
\int_{a}^{b}\left(\int_{a}^{b}|F(x, y)| d v(y)\right) d v(x)=2 C(p, f, g ; v) \geq 0
\end{gathered}
$$

and

$$
\begin{aligned}
\int_{a}^{b}\left(\int_{a}^{b}|F(x, y)| d u(y)\right) d v(x) & =\int_{a}^{b}\left(\int_{a}^{b}|F(x, y)| d v(y)\right) d u(x)= \\
& =C(p, f, g ; u, v) \geq 0 .
\end{aligned}
$$

Utilising inequality (19) we have

$$
\begin{aligned}
{[2 C(p, f, g ; h)]^{2} } & \leq[2 C(p, f, g ; u)]^{1 / 2}[2 C(p, f, g ; v)]^{1 / 2} \times \\
& \times[C(p, f, g ; u, v)]^{1 / 2}[C(p, f, g ; u, v)]^{1 / 2}
\end{aligned}
$$

which is clearly equivalent to 26].

For the complex-valued functions $p, f, g$ and $h, \ell$ defined on the interval $[a, b]$ we define the following (CBS)-type functionals

$$
\begin{aligned}
B(p, f, g ; h, \ell) & :=\int_{a}^{b} p|f|^{2} d h \int_{a}^{b} p|g|^{2} d \ell+\int_{a}^{b} p|g|^{2} d h \int_{a}^{b} p|f|^{2} d \ell- \\
& -\int_{a}^{b} p f \bar{g} d h \int_{a}^{b} p f \bar{g} d \ell-\int_{a}^{b} p \bar{f} g d h \int_{a}^{b} p \bar{f} g d \ell
\end{aligned}
$$

and

$$
B(p, f, g ; h):=\frac{1}{2} B(p, f, g ; h, h)=
$$




$$
\begin{aligned}
& =\int_{a}^{b} p|f|^{2} d h \int_{a}^{b} p|g|^{2} d h- \\
& -\frac{1}{2}\left[\left(\int_{a}^{b} p f \bar{g} d h\right)^{2}+\left(\int_{a}^{b} p \bar{f} g d h\right)^{2}\right] .
\end{aligned}
$$

If $p$ is nonnegative and $h$ is real-valued, then

$$
\left(\int_{a}^{b} p \bar{f} g d h\right)^{2}=\overline{\left(\int_{a}^{b} p f \bar{g} d h\right)^{2}}
$$

which implies that

$$
B(p, f, g ; h)=\int_{a}^{b} p|f|^{2} d h \int_{a}^{b} p|g|^{2} d h-\operatorname{Re}\left(\int_{a}^{b} p f \bar{g} d h\right)^{2} .
$$

Also, if $p$ is nonnegative and $f, g$ are real-valued, then

$$
B(p, f, g ; h)=\int_{a}^{b} p f^{2} d h \int_{a}^{b} p g^{2} d h-\left(\int_{a}^{b} p f g d h\right)^{2} .
$$

The following result also holds.

Theorem 5. Let $f, g:[a, b] \rightarrow \mathbb{C}$ be continuous on $[a, b]$. If $h:[a, b] \rightarrow$ $\rightarrow \mathbb{C}$ is an $S$-dominated function by the pair $(u, v)$, which are monotonic nondecreasing on $[a, b]$, then for any continuos nonnegative function $p$ : $[a, b] \rightarrow[0, \infty)$ we have

$$
|B(p, f, g ; h)|^{2} \leq \frac{1}{2} B(p, f, g ; u, v)[B(p, f, g ; u)]^{1 / 2}[B(p, f, g ; v)]^{1 / 2} .
$$

Proof. Define the function $F:[a, b] \times[a, b] \rightarrow \mathbb{R}$ by

$$
\begin{aligned}
F(x, y) & :=p(x) p(y)|f(x) \overline{g(y)}-g(x) \overline{f(y)}|^{2}= \\
& =p(y) p(x)\left[|f(x)|^{2}|g(y)|^{2}+|f(y)|^{2}|g(x)|^{2}-\right. \\
& -f(x) \overline{g(x)} f(y) \overline{g(y)}-\overline{f(x)} g(x) \overline{f(y)} g(y)] .
\end{aligned}
$$


We observe that, since $p$ is nonnegative, then $F(x, y) \geq 0$ for any $x, y \in$ $\in[a, b]$. The function $F$ is also continuous on the rectangle $[a, b] \times[a, b]$.

By simple calculation with the Riemann-Stieltjes integral we have

$$
\begin{gathered}
\int_{a}^{b}\left(\int_{a}^{b} F(x, y) d h(y)\right) d h(x)=2 B(p, f, g ; h), \\
\int_{a}^{b}\left(\int_{a}^{b}|F(x, y)| d u(y)\right) d u(x)=2 B(p, f, g ; u) \geq 0, \\
\int_{a}^{b}\left(\int_{a}^{b}|F(x, y)| d v(y)\right) d v(x)=2 B(p, f, g ; v) \geq 0
\end{gathered}
$$

and

$$
\begin{aligned}
\int_{a}^{b}\left(\int_{a}^{b}|F(x, y)| d u(y)\right) d v(x) & =\int_{a}^{b}\left(\int_{a}^{b}|F(x, y)| d v(y)\right) d u(x)= \\
& =B(p, f, g ; u, v) \geq 0 .
\end{aligned}
$$

Utilising the inequality 19 we have

$$
\begin{aligned}
{[2 B(p, f, g ; h)]^{2} } & \leq[2 B(p, f, g ; u)]^{1 / 2}[2 B(p, f, g ; v)]^{1 / 2} \times \\
& \times[B(p, f, g ; u, v)]^{1 / 2}[B(p, f, g ; u, v)]^{1 / 2}
\end{aligned}
$$

which is clearly equivalent to 30 .

5. Applications for Selfadjoint Operators. We denote by $\mathcal{B}(H)$ the Banach algebra of all bounded linear operators on a complex Hilbert space $(H ;\langle\cdot, \cdot\rangle)$. Let $A \in \mathcal{B}(H)$ be selfadjoint and let $\varphi_{\lambda}$ be defined for all $\lambda \in \mathbb{R}$ as follows

$$
\varphi_{\lambda}(s):=\left\{\begin{array}{l}
1, \text { for }-\infty<s \leq \lambda, \\
0, \text { for } \lambda<s<+\infty .
\end{array}\right.
$$

Then for every $\lambda \in \mathbb{R}$ the operator

$$
E_{\lambda}:=\varphi_{\lambda}(A)
$$

is a projection which reduces $A$. 
The properties of these projections are collected in the following fundamental result concerning the spectral representation of bounded selfadjoint operators in Hilbert spaces, see for instance [58, p. 256]:

Let $A$ be a bounded selfadjoint operator on the Hilbert space $H$.

$$
\text { Denote } \mathrm{m}=\min \{\lambda \mid \lambda \in \operatorname{Sp}(\mathrm{A})\}=: \min \operatorname{Sp}(\mathrm{A}) \text { and }
$$

$$
M=\max \{\lambda \mid \lambda \in \operatorname{Sp}(A)\}=: \max \operatorname{Sp}(A) .
$$

Then there exists a family of projections $\left\{E_{\lambda}\right\}_{\lambda \in \mathbb{R}}$, called the spectral family of $A$, with the following properties:

a) $E_{\lambda} \leq E_{\lambda^{\prime}}$ for $\lambda \leq \lambda^{\prime}$;

b) $E_{m-0}=0, E_{M}=I$ and $E_{\lambda+0}=E_{\lambda}$ for all $\lambda \in \mathbb{R}$.

We have the representation

$$
A=\int_{m-0}^{M} \lambda d E_{\lambda}
$$

More generally, for any continuous complex-valued function $\varphi$ defined on $\mathbb{R}$ and for any $\varepsilon>0$ there exists a $\delta>0$ such that

$$
\left\|\varphi(A)-\sum_{k=1}^{n} \varphi\left(\lambda_{k}^{\prime}\right)\left[E_{\lambda_{k}}-E_{\lambda_{k-1}}\right]\right\| \leq \varepsilon
$$

whenever

$$
\left\{\begin{array}{l}
\lambda_{0}<m=\lambda_{1}<\ldots<\lambda_{n-1}<\lambda_{n}=M, \\
\lambda_{k}-\lambda_{k-1} \leq \delta \text { for } 1 \leq k \leq n \\
\lambda_{k}^{\prime} \in\left[\lambda_{k-1}, \lambda_{k}\right] \text { for } 1 \leq k \leq n
\end{array}\right.
$$

this means that

$$
\varphi(A)=\int_{m-0}^{M} \varphi(\lambda) d E_{\lambda},
$$

where the integral is of the Riemann-Stieltjes type.

With the above assumptions for $A, E_{\lambda}$ and $\varphi$ we have the representations

$$
\varphi(A) x=\int_{m-0}^{M} \varphi(\lambda) d E_{\lambda} x \text { for all } x \in H
$$


and

$$
\langle\varphi(A) x, y\rangle=\int_{m-0}^{M} \varphi(\lambda) d\left\langle E_{\lambda} x, y\right\rangle \quad \text { for all } x, y \in H .
$$

In particular,

$$
\langle\varphi(A) x, x\rangle=\int_{m-0}^{M} \varphi(\lambda) d\left\langle E_{\lambda} x, x\right\rangle \quad \text { for all } x \in H .
$$

Moreover, we have the equality

$$
\|\varphi(A) x\|^{2}=\int_{m-0}^{M}|\varphi(\lambda)|^{2} d\left\|E_{\lambda} x\right\|^{2} \quad \text { for all } x \in H .
$$

Utilising Theorem 1 we can prove easily the following Schwarz type inequality:

Proposition 2. Let $A$ be a bounded selfadjoint operator on the Hilbert space $H$. Denote $m:=\min \{\lambda \mid \lambda \in \operatorname{Sp}(A)\}=\min \operatorname{Sp}(A)$ and $M:=$ $:=\max \{\lambda \mid \lambda \in \operatorname{Sp}(A)\}=\max \operatorname{Sp}(A)$. If $f: \mathbb{R} \rightarrow \mathbb{C}$ is a continuous function on $[m, M]$, then we have the inequality

$$
|\langle f(A) x, y\rangle|^{2} \leq\langle|f(A)| x, x\rangle\langle|f(A)| y, y\rangle
$$

for any $x, y \in H$.

Proof. Assume $\varepsilon>0$ and for fixed $x, y \in H$ define the functions $h, u, v$ : $[m-\varepsilon, M] \rightarrow \mathbb{C}$ given by

$$
h(t):=\left\langle E_{t} x, y\right\rangle, u(t):=\left\langle E_{t} x, x\right\rangle \text { and } v(t):=\left\langle E_{t} y, y\right\rangle
$$

where $\left\{E_{\lambda}\right\}_{\lambda \in \mathbb{R}}$ is the spectral family of the bounded selfadjoint operator A.

For $t, s \in[m-\varepsilon, M]$ with $t>s$ by utilizing the Schwarz inequality for nonnegative operators $P$

$$
|\langle P x, y\rangle|^{2} \leq\langle P x, x\rangle\langle P y, y\rangle,
$$

we have

$$
\begin{gathered}
|h(t)-h(s)|^{2}=\left|\left\langle\left(E_{t}-E_{s}\right) x, y\right\rangle\right|^{2} \leq \\
\leq\left\langle\left(E_{t}-E_{s}\right) x, x\right\rangle\left\langle\left(E_{t}-E_{s}\right) y, y\right\rangle=(u(t)-u(s))(v(t)-v(s)),
\end{gathered}
$$


which shows that $h$ is $S$-dominated by the monotonic nondecreasing functions $(u, v)$ on $[m-\varepsilon, M]$.

Applying Theorem 1 to $f, h, u$ and $v$ on $[m-\varepsilon, M]$ we have

$$
\left|\int_{m-\varepsilon}^{M} f(t) d\left(\left\langle E_{t} x, y\right\rangle\right)\right|^{2} \leq \int_{m-\varepsilon}^{M}|f(t)| d\left(\left\langle E_{t} x, x\right\rangle\right) \int_{m-\varepsilon}^{M}|f(t)| d\left(\left\langle E_{t} y, y\right\rangle\right)
$$

for any $x, y \in H$.

Letting $\varepsilon \rightarrow 0+$ in $(33)$ and utilizing the representation of continuous functions of selfadjoint operators, we deduce the desired result (32).

For continuous functions $p, f, g$, the selfadjoint operator $A$ and $x, y \in$ $\in H$ we define the functionals

$$
\begin{aligned}
C(p, f, g ; A, x, y) & :=\langle p(A) x, y\rangle\langle p(A) f(A) g(A) x, y\rangle- \\
& -\langle p(A) f(A) x, y\rangle\langle p(A) g(A) x, y\rangle \\
C(p, f, g ; A, x) & :=C(p, f, g ; A, x, x)= \\
& =\langle p(A) x, x\rangle\langle p(A) f(A) g(A) x, x\rangle- \\
& -\langle p(A) f(A) x, x\rangle\langle p(A) g(A) x, x\rangle
\end{aligned}
$$

and

$$
D(p, f, g ; A, x, y):=
$$

$:=\langle p(A) x, x\rangle\langle p(A) f(A) g(A) y, y\rangle+\langle p(A) y, y\rangle\langle p(A) f(A) g(A) x, x\rangle-$

- $\langle p(A) g(A) x, x\rangle\langle p(A) f(A) y, y\rangle-\langle p(A) g(A) y, y\rangle\langle p(A) f(A) x, x\rangle$.

The following result holds:

Proposition 3. Let $A$ be a bounded selfadjoint operator on the Hilbert space $H$. Denote $m:=\min \{\lambda \mid \lambda \in \operatorname{Sp}(A)\}=\min \operatorname{Sp}(A)$ and $M:=$ $:=\max \{\lambda \mid \lambda \in \operatorname{Sp}(A)\}=\max \operatorname{Sp}(A)$. Assume that $f, g: \mathbb{R} \rightarrow \mathbb{R}$ are continuous and synchronous functions on $[m, M]$ and $p: \mathbb{R} \rightarrow \mathbb{R}$ is a nonnegative continuous function on $[m, M]$. Then for any $x, y \in H$ we have

$$
\begin{aligned}
& |C(p, f, g ; A, x, y)|^{2} \leq \\
& \leq \frac{1}{2} D(p, f, g ; A, x, y)[C(p, f, g ; A, x)]^{1 / 2}[C(p, f, g ; A, y)]^{1 / 2} .
\end{aligned}
$$


The proof is similar to the one from Proposition 2 may be obtained by utilizing the integral inequality from Theorem 4. The details are omitted.

A simpler version of the above inequality $(34)$ is as follows:

Corollary 2. Let the assumptions of Proposition 3 for $A, f$ and $g$ be valid. Then for any $x, y \in H$ with $\|x\|=\|y\|=1$ we have

$$
\begin{aligned}
& |\langle x, y\rangle\langle f(A) g(A) x, y\rangle-\langle f(A) x, y\rangle\langle g(A) x, y\rangle|^{2} \leq \\
& \leq \frac{1}{2}[\langle f(A) g(A) y, y\rangle+\langle f(A) g(A) x, x\rangle- \\
& -\langle g(A) x, x\rangle\langle f(A) y, y\rangle-\langle g(A) y, y\rangle\langle f(A) x, x\rangle] \times \\
& \times[\langle f(A) g(A) x, x\rangle-\langle f(A) x, x\rangle\langle g(A) x, x\rangle]^{1 / 2} \times \\
& \times[\langle f(A) g(A) y, y\rangle-\langle f(A) y, y\rangle\langle g(A) y, y\rangle]^{1 / 2} .
\end{aligned}
$$

Remark 1. If we take, as an example, $f(t)=t^{p}$ and $g(t)=t^{q}$ for $p, q>0$ then for any positive operator $A$ we have from (35) the inequality

$$
\begin{aligned}
& \left|\langle x, y\rangle\left\langle A^{p+q} x, y\right\rangle-\left\langle A^{p} x, y\right\rangle\left\langle A^{q} x, y\right\rangle\right|^{2} \leq \\
& \leq \frac{1}{2}\left[\left\langle A^{p+q} y, y\right\rangle+\left\langle A^{p+q} x, x\right\rangle-\left\langle A^{q} x, x\right\rangle\left\langle A^{p} y, y\right\rangle-\left\langle A^{q} y, y\right\rangle\left\langle A^{p} x, x\right\rangle\right] \times \\
& \times\left[\left\langle A^{p+q} x, x\right\rangle-\left\langle A^{p} x, x\right\rangle\left\langle A^{q} x, x\right\rangle\right]^{1 / 2}\left[\left\langle A^{p+q} y, y\right\rangle-\left\langle A^{p} y, y\right\rangle\left\langle A^{q} y, y\right\rangle\right]^{1 / 2},
\end{aligned}
$$

for any $x, y \in H$ with $\|x\|=\|y\|=1$.

6. Applications for Unitary Operators. Let $(H,\langle\cdot, \cdot\rangle)$ be a complex Hilbert space. We recall that the bounded linear operator $U$ : $H \rightarrow H$ on $H$ is unitary iff $U^{*}=U^{-1}$.

It is well known that (see for instance [58, pp. 275-276]), if $U$ is a unitary operator, then there exists a family of projections $\left\{E_{\lambda}\right\}_{\lambda \in[0,2 \pi]}$, called the spectral family of $U$ with the following properties:

a) $E_{\lambda} \leq E_{\mu}$ for $0 \leq \lambda \leq \mu \leq 2 \pi$;

b) $E_{0}=0$ and $E_{2 \pi}=1_{H}$ (the identity operator on $H$ );

c) $E_{\lambda+0}=E_{\lambda}$ for $0 \leq \lambda<2 \pi$;

d) $U=\int_{0}^{2 \pi} e^{i \lambda} d E_{\lambda}$ where the integral is of the Riemann-Stieltjes type.

Moreover, if $\left\{F_{\lambda}\right\}_{\lambda \in[0,2 \pi]}$ is a family of projections satisfying the requirements a)-d) above for the operator $U$, then $F_{\lambda}=E_{\lambda}$ for all $\lambda \in$ $\in[0,2 \pi]$. 
Also, for each continuous complex-valued function $f: \mathcal{C}(0,1) \rightarrow \mathbb{C}$ on the complex unit circle $\mathcal{C}(0,1)$, we have

$$
f(U)=\int_{0}^{2 \pi} f\left(e^{i \lambda}\right) d E_{\lambda},
$$

where the integral is taken in the Riemann-Stieltjes sense.

In particular, we have the equalities

$$
\begin{gathered}
f(U) x=\int_{0}^{2 \pi} f\left(e^{i \lambda}\right) d E_{\lambda} x, \\
\langle f(U) x, y\rangle=\int_{0}^{2 \pi} f\left(e^{i \lambda}\right) d\left\langle E_{\lambda} x, y\right\rangle
\end{gathered}
$$

and

$$
\|f(U) x\|^{2}=\int_{0}^{2 \pi}\left|f\left(e^{i \lambda}\right)\right|^{2} d\left\|E_{\lambda} x\right\|^{2},
$$

for any $x, y \in H$.

Proposition 4. Let $U$ be a unitary operator on a Hilbert space $H$. Then for each continuous complex-valued function $f: \mathcal{C}(0,1) \rightarrow \mathbb{C}$ on the complex unit circle $\mathcal{C}(0,1)$, we have

$$
|\langle f(U) x, y\rangle|^{2} \leq\langle|f(U)| x, x\rangle\langle|f(U)| y, y\rangle
$$

for any $x, y \in H$.

Proof. Let $\left\{E_{\lambda}\right\}_{\lambda \in[0,2 \pi]}$ be the spectral family of the unitary operator $U$. For fixed $x, y \in H$ define the functions $h, u, v:[0,2 \pi] \rightarrow \mathbb{C}$ given by

$$
h(t):=\left\langle E_{t} x, y\right\rangle, u(t):=\left\langle E_{t} x, x\right\rangle \text { and } v(t):=\left\langle E_{t} y, y\right\rangle .
$$

For $t, s \in[0,2 \pi]$, with $t>s$, by utilizing the Schwarz inequality for nonnegative operators $P$

$$
|\langle P x, y\rangle|^{2} \leq\langle P x, x\rangle\langle P y, y\rangle,
$$

we have

$$
\begin{gathered}
|h(t)-h(s)|^{2}=\left|\left\langle\left(E_{t}-E_{s}\right) x, y\right\rangle\right|^{2} \leq \\
\leq\left\langle\left(E_{t}-E_{s}\right) x, x\right\rangle\left\langle\left(E_{t}-E_{s}\right) y, y\right\rangle=(u(t)-u(s))(v(t)-v(s)),
\end{gathered}
$$


which shows that $h$ is $S$-dominated by the monotonic nondecreasing functions $(u, v)$ on $[0,2 \pi]$.

Applying Theorem 1 to $f\left(e^{i t}\right), h, u$ and $v$ on $[0,2 \pi]$ we have

$$
\begin{gathered}
\left|\int_{0}^{2 \pi} f\left(e^{i t}\right) d\left(\left\langle E_{t} x, y\right\rangle\right)\right|^{2} \leq \\
\leq \int_{0}^{2 \pi}\left|f\left(e^{i t}\right)\right| d\left(\left\langle E_{t} x, x\right\rangle\right) \int_{0}^{2 \pi}\left|f\left(e^{i t}\right)\right| d\left(\left\langle E_{t} y, y\right\rangle\right)
\end{gathered}
$$

for any $x, y \in H$.

Utilising the representation of continuous functions of unitary operators, we deduce the desired result (36).

For the complex-valued functions $f, g$ defined on the complex unit circle $\mathcal{C}(0,1)$ and the unitary operator $U$ on the Hilbert space $H$ we define the following functionals:

$D(f, g ; U, x, y):=$

$:=\left\langle|f(U)|^{2} x, x\right\rangle\left\langle|g(U)|^{2} y, y\right\rangle+\left\langle|g(U)|^{2} x, x\right\rangle\left\langle|f(U)|^{2} y, y\right\rangle-$

$-\langle f(U) \bar{g}(U) x, x\rangle\langle f(U) \bar{g}(U) y, y\rangle-\langle\bar{f}(U) g(U) x, x\rangle\langle\bar{f}(U) g(U) y, y\rangle$,

$B(f, g ; U, x, y):=\left\langle|f(U)|^{2} x, y\right\rangle\left\langle|g(U)|^{2} x, y\right\rangle-$

$-\frac{1}{2}\left[\langle f(U) \bar{g}(U) x, y\rangle^{2}+\langle\bar{f}(U) g(U) x, y\rangle^{2}\right]$

and

$B(f, g ; U, x):=B(f, g ; U, x, x)=$

$$
=\left\langle|f(U)|^{2} x, x\right\rangle\left\langle|g(U)|^{2} x, x\right\rangle-\operatorname{Re}\langle f(U) \bar{g}(U) x, x\rangle^{2},
$$

where $x, y \in H$.

Proposition 5. Let $U$ be a unitary operator on a Hilbert space $H$. Then for every continuous complex-valued functions $f, g: \mathcal{C}(0,1) \rightarrow \mathbb{C}$ on the complex unit circle $\mathcal{C}(0,1)$, we have

$$
|B(f, g ; U, x, y)|^{2} \leq \frac{1}{2} D(f, g ; U, x, y)[B(f, g ; U, x)]^{1 / 2}[B(f, g ; U, y)]^{1 / 2}
$$

for any $x, y \in H$. 
The proof follows from Theorem 5 applied to the functions $f\left(e^{i t}\right)$, $g\left(e^{i t}\right), p(t)=1, h(t):=\left\langle E_{t} x, y\right\rangle, u(t):=\left\langle E_{t} x, x\right\rangle$ and $v(t):=\left\langle E_{t} y, y\right\rangle$ where $\left\{E_{\lambda}\right\}_{\lambda \in[0,2 \pi]}$ is the spectral family of the unitary operator $U$ and $t \in[0,2 \pi]$. The details are omitted.

Acknowledgment. The author would like to thank the anonymous referees for valuable suggestions that have been implemented in the final version of the paper.

\section{References}

[1] Dragomir S. S. The Ostrowski inequality for mappings of bounded variation. Bull. Austral. Math. Soc., 1999, vol. 60, pp. 495-826.

[2] Cerone P., Dragomir S. S. and Pearce C. E. M. A generalised trapezoid inequality for functions of bounded variation. Turk. J. Math., 2000, vol. 24, pp. $147-163$.

[3] Barnett N. S., Cheung W. S., Dragomir S. S. and Sofo A. Ostrowski and trapezoid type inequalities for the Stieltjes integral with Lipschitzian integrands or integrators. Comput. Math. Appl., 2009, vol. 57, pp. 195201.

[4] Barnett N. S. and Dragomir S. S. A perturbed trapezoid inequality in terms of the fourth derivative. Korean J. Comput. Appl. Math., 2002, vol. 9, pp. $45-60$.

[5] Barnett N. S. and Dragomir S. S. Perturbed version of a general trapezoid inequality. Inequality theory and applications, 2003, vol. 3, pp. 1-12.

[6] Barnett N. S. and Dragomir S. S. A perturbed trapezoid inequality in terms of the third derivative and applications. Inequality theory and applications, 2007, vol. 5, pp. 1-11.

[7] Barnett N. S., Dragomir S. S. and Gomm I. A companion for the Ostrowski and the generalised trapezoid inequalities. Math. Comput. Modelling, 2009, vol. 50, pp. 179-187.

[8] Cerone P. and Dragomir S. S. Midpoint-type rules from an inequalities point of view. Handbook of analytic-computational methods in applied mathematics, 2000, pp. 135-200.

[9] Cerone P. and Dragomir S. S. Trapezoidal-type rules from an inequalities point of view. Handbook of analytic-computational methods in applied mathematics, 2000, pp. 65-134.

[10] Cheng X. L. and Sun J. A note on the perturbed trapezoid inequality. J. Inequal. Pure Appl. Math., 2002, vol. 3, Article 29, 7 pp. (electronic). 
[11] Dragomir S. S. On the trapezoid quadrature formula and applications. Kragujevac J. Math., 2001, vol. 23, pp. 25-36.

[12] Dragomir S. S. Some inequalities of midpoint and trapezoid type for the Riemann-Stieltjes integral. Nonlinear Anal., 2001, vol. 47, pp. 2333-2340.

[13] Dragomir S. S. Improvements of Ostrowski and generalised trapezoid inequality in terms of the upper and lower bounds of the first derivative. Tamkang J. Math., 2003, vol. 34, pp. 213-222.

[14] Dragomir S. S. Refinements of the generalised trapezoid and Ostrowski inequalities for functions of bounded variation. Arch. Math., 2008, vol. 91, pp. $450-460$.

[15] Dragomir S. S., Cho Y. J. and Kim Y. H. On the trapezoid inequality for the Riemann-Stieltjes integral with Hölder continuous integrands and bounded variation integrators. Inequality theory and applications, 2007, vol. 5, pp. 71-79.

[16] Dragomir S. S. and Mcandrew A. On trapezoid inequality via a Grüss type result and applications. Tamkang J. Math., 2000, vol. 31, pp. 193-201.

[17] Dragomir S. S., Pečarić J. and Wang S. The unified treatment of trapezoid, Simpson, and Ostrowski type inequality for monotonic mappings and applications. Math. Comput. Modelling, 2000, vol. 31, pp. 61-70.

[18] Gunawan H. A note on Dragomir-McAndrew's trapezoid inequalities. Tamkang J. Math., 2002, vol. 33, pp. 241-244.

[19] Liu Z. Some inequalities of perturbed trapezoid type. J. Inequal. Pure Appl. Math., 2006, vol. 7, Article 47, 9 pp.

[20] Liu W. J., Xue Q. L. and Dong J. W. New generalization of perturbed trapezoid, mid-point inequalities and applications. Int. J. Pure Appl. Math., 2007, vol. 41, pp. 761-768.

[21] Kechriniotis A. I. and Assimakis N. D. Generalizations of the trapezoid inequalities based on a new mean value theorem for the remainder in Taylor's formula. J. Inequal. Pure Appl. Math., 2006, vol. 7, Article 90, 13 pp. (electronic).

[22] Mercer P. R. Hadamard's inequality and trapezoid rules for the RiemannStieltjes integral. J. Math. Anal. Appl., 2008, vol. 344, pp. 921-926.

[23] Mercer A. McD. On perturbed trapezoid inequalities. J. Inequal. Pure Appl. Math., 2006, vol. 7, Article 118, 7 pp. (electronic).

[24] Pachpatte B. G. A note on a trapezoid type integral inequality. Bull. Greek Math. Soc., 2004, vol. 49, pp. 85-90.

[25] Ujević N. Perturbed trapezoid and mid-point inequalities and applications. Soochow J. Math., 2003, vol. 29, pp. 249-257. 
[26] Ujević N. On perturbed mid-point and trapezoid inequalities and applications. Kyungpook Math. J., 2003, vol. 43, pp. 327-334.

[27] Ujević N. Error inequalities for a generalized trapezoid rule. Appl. Math. Lett., 2006, vol. 19, pp. 32-37.

[28] Dragomir S. S. On the Ostrowski's integral inequality for mappings with bounded variation and applications. Math. Ineq. Appl., 2001, vol. 4, pp. 3340. Preprint, RGMIA Res. Rep. Coll., 1999, vol. 2, no. 1, Article 7.

[29] Acu A. M., Baboş A. and Sofonea F. The mean value theorems and inequalities of Ostrowski type. Sci. Stud. Res. Ser. Math. Inform., 2001, vol. 21, pp. 5-16.

[30] Acu A. M. and Sofonea F. On an inequality of Ostrowski type. J. Sci. Arts., 2011, vol. 3(16), pp. 281-287.

[31] Ahmad F., Barnett N. S. and Dragomir S. S. New weighted Ostrowski and Čebyšev type inequalities. Nonlinear Anal., 2009, vol. 71, pp. e1408-e1412.

[32] Alomari M. W. A companion of Ostrowski's inequality with applications. Transylv. J. Math. Mech., 2011, vol. 3, pp. 9-14.

[33] Alomari M. W., Darus M., Dragomir S. S. and Cerone P. Ostrowski type inequalities for functions whose derivatives are s-convex in the second sense. Appl. Math. Lett., 2010, vol. 23, pp. 1071-1076.

[34] Anastassiou G. A. Ostrowski type inequalities. Proc. Amer. Math. Soc., 1995, vol. 123, pp. 3775-3781.

[35] Anastassiou G. A. Univariate Ostrowski inequalities, revisited. Monatsh. Math., 2002, vol. 135, pp. 175-189.

[36] Anastassiou G. A. Ostrowski inequalities for cosine and sine operator functions. Mat. Vestnik, 2012, vol. 64, pp. 336-346.

[37] Anastassiou G. A. Multivariate right fractional Ostrowski inequalities. J. Appl. Math. Inform., 2012, vol. 30, pp. 445-454.

[38] Anastassiou G. A. Univariate right fractional Ostrowski inequalities. Cubo, 2012, vol. 14, pp. 1-7.

[39] Cerone P., Cheung W. S. and Dragomir S. S. On Ostrowski type inequalities for Stieltjes integrals with absolutely continuous integrands and integrators of bounded variation. Comput. Math. Appl., 2007, vol. 54, pp. 183-191.

[40] Dragomir S. S. On the mid-point quadrature formula for mappings with bounded variation and applications. Kragujevac J. Math., 2000, vol. 22, pp. 13-19.

[41] Dragomir S. S. Some inequalities for continuous functions of selfadjoint operators in Hilbert aces. Acta Math. Vietnamica, 2014, vol. 39, pp. 287303. 
[42] Liu Z. Some Ostrowski type inequalities and applications. Vietnam J. Math., 2009, vol. 37, pp. 15-22.

[43] Liu Z. Some companions of an Ostrowski type inequality and applications. J. Inequal. Pure Appl. Math., 2009, vol. 10, Article 52, 12 pp.

[44] Liu Z. A note on Ostrowski type inequalities related to some s-convex functions in the second sense. Bull. Korean Math. Soc., 2012, vol. 49, pp. 775785.

[45] Liu Z. A sharp general Ostrowski type inequality. Bull. Aust. Math. Soc., 2011, vol. 83, pp. 189-209.

[46] Masjed-Jamei M. and Dragomir S. S. A new generalization of the Ostrowski inequality and applications. Filomat, 2011, vol. 25, pp. 115-123.

[47] Pachpatte B. G. New inequalities of Ostrowski and trapezoid type for n-time differentiable functions. Bull. Korean Math. Soc., 2004, vol. 41, pp. 633-639.

[48] Park J. On the Ostrowskilike type integral inequalities for mappings whose second derivatives are $s^{*}$-convex. Far East J. Math. Sci., 2012, vol. 67, pp. 21-35.

[49] Park J. Some Ostrowskilike type inequalities for differentiable real $(\alpha, m)$ convex mappings. Far East J. Math. Sci., 2012, vol. 61, pp. 75-91.

[50] Sarikaya Z. On the Ostrowski type integral inequality. Acta Math. Univ. Comenian. (N. S.), 2010, vol. 79, pp. 129-134.

[51] Sulaiman W. T. Some new Ostrowski type inequalities. J. Appl. Funct. Anal., 2012, vol. 7, pp. 102-107.

[52] Tseng K. L. Improvements of the Ostrowski integral inequality for mappings of bounded variation II. Appl. Math. Comput., 2012, vol. 218, pp. 58415847.

[53] Tseng K. L., Hwang S. R., Yang G. S. and Chou Y. M. Improvements of the Ostrowski integral inequality for mappings of bounded variation I. Appl. Math. Comput., 2010, vol. 217, pp. 2348-2355.

[54] Vong S. W. A note on some Ostrowskilike type inequalities. Comput. Math. Appl., 2011, vol. 62, pp. 532-535.

[55] Wu Q. and Yang S. A note to Ujević's generalization of Ostrowski's inequality. Appl. Math. Lett., 2005, vol. 18, pp. 657-665.

[56] Wu Y. and Wang Y. On the optimal constants of Ostrowskilike inequalities involving $n$ knots. Appl. Math. Comput., 2013, vol. 219, pp. 7789-7794.

[57] Xiao Y. X. Remarks on Ostrowskilike inequalities.Appl. Math. Comput., 2012, vol. 219, pp. 1158-1162. 
[58] Helmberg G. Introduction to spectral theory in Hilbert space. John Wiley \& Sons, Inc., New York, 1969.

Received April 14, 2015.

In revised form, June 18, 2015.

Victoria University

PO Box 14428, Melbourne City, MC 8001, Australia

E-mail: sever.dragomir@vu.edu.au 